\title{
Towards Parallel Constraint-Based Local Search with the X10 Language
}

\author{
Danny Munera ${ }^{1}$, Daniel Diaz ${ }^{1}$, and Salvador Abreu ${ }^{2}$ \\ 1 University of Paris 1-Sorbonne, France \\ Danny.Munera@malix.univ-paris1.fr, Daniel.Diaz@univ-paris1.fr \\ 2 Universidade de Évora and CENTRIA, Portugal \\ spa@di.uevora.pt
}

\begin{abstract}
In this study, we started to investigate how the Partitioned Global Address Space (PGAS) programming language X10 would suit the implementation of a Constraint-Based Local Search solver. We wanted to code in this language because we expect to gain from its ease of use and independence from specific parallel architectures. We present our implementation strategy, and quest for different sources of parallelism. We discuss the algorithms, their implementations and present a performance evaluation on a representative set of benchmarks.
\end{abstract}

\section{Introduction}

Constraint Programming has been successfully used to model and solve many real-life problems in diverse areas such as planning, resource allocation, scheduling and product line modeling $[18,19]$. Classically, constraint satisfaction problems (CSPs) may be solved exhaustively by complete methods which are capable of finding all solutions, and therefore to determine whether any solutions exist. However efficient these solvers may be, a significant class of problems remains out of reach because of exponential growth of the search space, which must be exhaustively explored. Another approach to solving CSPs entails giving up completeness and resorting to (meta-) heuristics which will guide the process of searching for solutions to the problem. Solvers in this class make choices which limit the search space which actually gets visited, enough so to make problems tractable. For instance a complete solver for the magic squares benchmark will fail for problems larger than $15 \times 15$ whereas a local search method will easily solve a $100 \times 100$ problem instance, within similar memory and CPU time bounds. On the other hand, a local search procedure may not be able to find a solution, even when one exists.

However, it is unquestionable that the more computational resources are available, the more complex the problems that may be solved. We would therefore like to be able to tap into the forms of augmented computational power which are actually available, as conveniently as feasible. This requires taming various forms of explicitly parallel architectures.

Present-day parallel computational resources include increasingly multi-core processors, General Purpose Graphic Processing Units (GPGPUs), computer 
clusters and grid computing platforms. Each of these forms requires a different programming model and the use of specific software tools, the combination of which makes software development even more difficult.

The foremost software platforms used for parallel programming include POSIX Threads [2] and OpenMP [17] for shared-memory multiprocessors and multicore CPUs, MPI [23] for distributed-memory clusters or CUDA [16] and OpenCL [12] for massively parallel architectures such as GPGPUs. This diversity is a challenge from the programming language design standpoint, and a few proposals have emerged that try to simultaneously address the multiplicity of parallel computational architectures.

Several modern language designs are built around the Partitioned Global Address Space (PGAS) memory model, as is the case with X10 [21], Unified Parallel C [7] or Chapel [10]. Many of these languages propose abstractions which capture the several forms in which multiprocessors can be organized. Other, less radical, approaches consist in supplying a library of inter-process communication which relies on and uses a PGAS model [14].

In our quest to find a scalable and architecture-independent implementation platform for our exploration of high-performance parallel constraint-based local search methods, we decided to experiment with one of the most promising newgeneration languages, X10 [21].

The remainder of this article is organized as follows: Section 2 discusses the PGAS Model and briefly introduces the X10 programming language. Section 3 introduces native X10 implementations exploiting different sources of parallelism of the Adaptive Search algorithm. Section 4 presents an evaluation of these implementations. A short conclusion ends the paper.

\section{X10 and the Partitioned Global Address Space (PGAS) model}

The current arrangement of tools to exploit parallelism in machines are strongly linked to the platform used. Two broad programming models stand out in this matter: distributed and shared memory models. For large distributed memory systems, like clusters and grid computing, the Message Passing Interface (MPI) [23] is a de-facto programming standard. The key idea in MPI is to decompose the computation over a collection of processes, each with its private memory space. These processes can communicate with each other through message passing, generally over a communication network.

With the recent growth of many-core architectures, the shared memory approach has grown in popularity. This model decomposes the computation in multiple threads of execution which share a common address space, communicating with each other by reading and writing shared variables. Actually, this is the model used by traditional programming tools like Fortran or $\mathrm{C}$ through libraries like pthreads [2] or OpenMP [17].

The PGAS model tries to combine the advantages of these two approaches: it extends shared memory to a distributed memory setting. The execution model 
allows having multiple processes (like MPI), multiple threads in a process (like OpenMP), or a combination thereof (see Figure 1). Ideally, the user would be allowed to decide how tasks get mapped to physical resources. X10 [21], Unified Parallel C [25] and Chapel [10] are examples of PGAS-enabled languages, but there are also PGAS-based IPC libraries such as GPI [14], for use in traditional programming languages. For the experiments described herein, we used the X10 language.

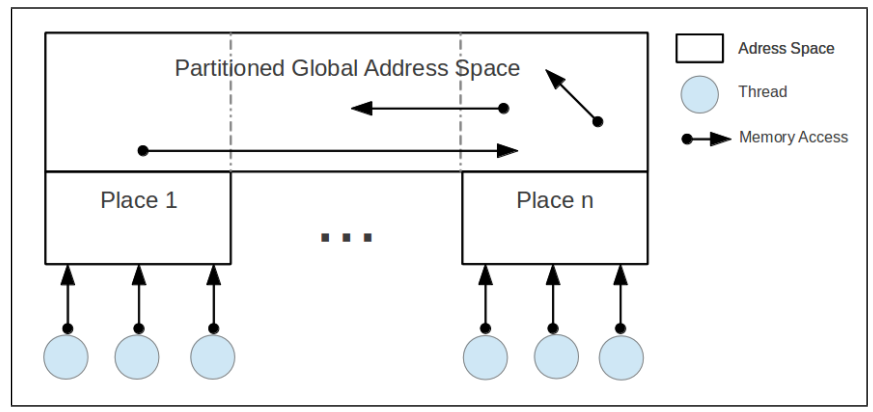

Fig. 1. PGAS Model

$\mathrm{X} 10$ [21] is a general-purpose language developed by IBM, which provides a PGAS variation: Asynchronous PGAS (APGAS). APGAS extends the PGAS model making it flexible, even in non-HPC platforms [20]. With this model X10 supports different levels of concurrency with simple language constructs.

There are two main abstractions in X10: places and activities. A place is the abstraction of a virtual shared-memory process, it has a coherent portion of the address space together with threads (activities) that operate on that memory. The X10 construct for creating a place in X10 is at, and is commonly used to create a place for each processing unit in the platform. An activity is the mechanism to abstract the single threads that perform computation within a place. Multiple activities may be simultaneously active in one place.

X10 implements the major components of the PGAS model, by the use of places and activities. However, the language includes other interesting tools with the goal of improving the abstraction level of the language. Synchronization is supported thanks to various operations such as finish, atomic and clock. The finish operation is used to wait for the termination of a set of activities, it behaves like a traditional barrier. The constructs atomic ensures exclusive access to a critical portion of code. Finally, the construct clock is the standard way to ensure the synchronization between activities or places. X10 supports the distributed array construct, which makes it possible to divide an array into subarrays which are mapped to available places. Doing this ensures a local access from each place to the related assigned sub-array. A detailed discussion of X10, including a tutorial, language specification and examples can be consulted at http://x10-lang.org/. 


\section{Native X10 Implementations of Adaptive Search}

In order to take advantage of parallelism it is necessary to identify the opportunities which exist within the Adaptive Search algorithm. In [5], the authors survey the state-of-the-art of the main parallel meta-heuristic strategies and discuss general design and implementation principles. They classify the decomposition of activities for parallel work in two main groups: functional parallelism and data parallelism. $^{1}$

On the one hand, in functional parallelism different tasks run on multiple compute instances across the same or different data-sets. On the other hand, data parallelism refers to the methods in which the problem domain or the associated search space is decomposed. A particular solution methodology is used to address the problem on each of the resulting components of the search space. This article reports on our experiments concerning both kinds of parallelism applied to the Adaptive Search method.

\subsection{Sequential Implementation}

Our first experiment with AS in X10 was to develop a sequential implementation corresponding to a specialized version of the Adaptive Search for permutation problems [15]. ${ }^{2}$

Figure 2 shows the class diagram of the basic X10 project. The class ASPermutSolver contains the Adaptive Search permutation specialized method implementation. This class inherits the basic functionality from a general implementation of the Adaptive Search solver (in class AdaptiveSearchSolver), which in turn inherits a very simple Local Search method implementation from the class LocalSearchSolver. This class is then specialized for different parallel approaches, which we experimented with. As we will see below, we experimented with two versions of Functional Parallelism (FP1 and FP2) and a Data Parallelism version (called Independent Multi-Walk, i.e. IMW).

Moreover, a simple CSP model is described in the class CSPModel, and specialized implementations of each CSP benchmark problem are contained in the classes PartitModel, MagicSquareModel, AllIntervallModel and CostasModel, which have all data structures and methods to implement the error function of each problem.

Listing 1.1 shows a simplified skeleton code of our X10 sequential implementation, based on Algorithm 1. The core of the Adaptive Search algorithm is implemented in the method solve. The solve method receives a CSPModel instance as parameter. On line 8, the CSP variables of the model are initialized with a random permutation. On the next line the total cost of the current

\footnotetext{
${ }^{1}$ Their relation is similar to that of AND- and OR-parallelism in the Logic Programming community.

${ }^{2}$ In a permutation problem, all $N$ variables have the same initial domain of size $N$ and are subject to an implicit all-different constraint. The associated algorithm is reported in the appendix.
} 


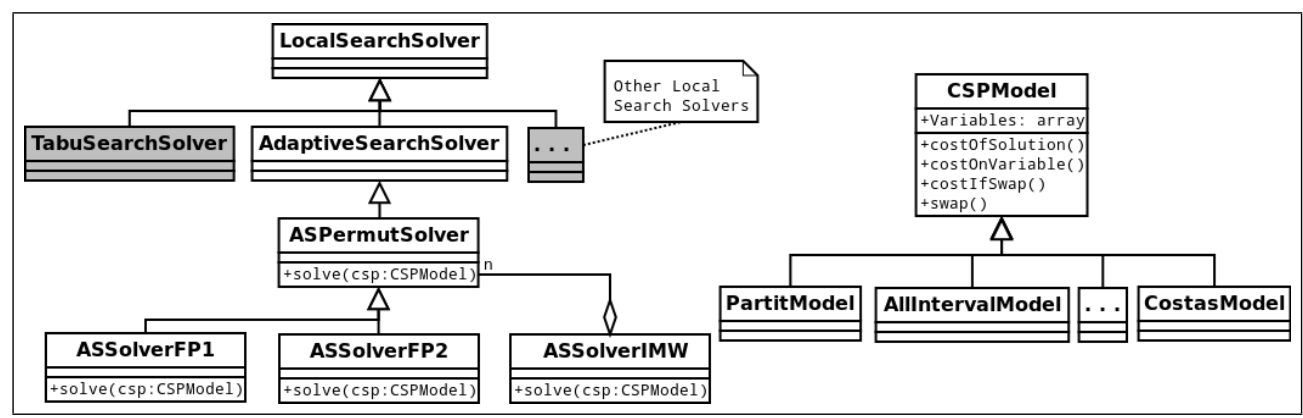

Fig. 2. X10 Class Diagram basic project

configuration is computed. The while instruction on line 10 corresponds to the main loop of the algorithm. The selectVarHighCost function (Line 12) selects the variable with the maximal error and saves the result in the maxI variable. The selectVarMinConflict function (Line 13) selects the best neighbor move from the highest cost variable $\operatorname{maxI}$, and saves the result in the minJ variable. Finally, if no local minimum is detected, the algorithm swaps the variables maxI and minJ (permutation problem) and computes the total cost of the resulting new configuration (Line 16). The solver function ends if the totalCost variable equals 0 or when the maximum number of iterations is reached.

Listing 1.1. Simplified AS X10 Sequential Implementation

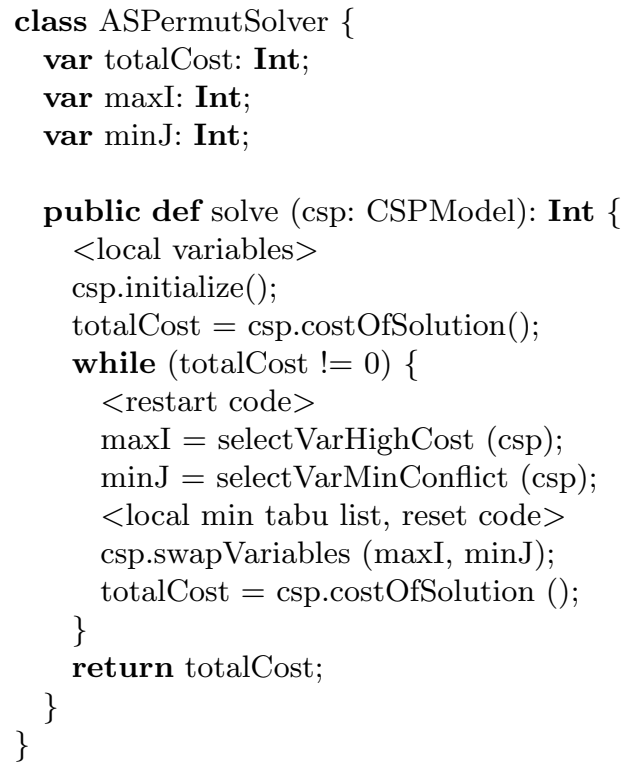




\subsection{Functional Parallel Implementation}

Functional parallelism is our first attempt to parallelize the Adaptive Search algorithm. The key aim for this implementation is to decompose the problem into different tasks, each task working in parallel on the same data. To achieve this objective it is necessary to change the inner loop of the sequential Adaptive Search algorithm.

In this experiment, we decided to change the structure of the selectVarHighCost function, because therein lies the most costly activities performed in the inner loop. The most important task performed by this function is to go through the variable array of the CSP model to compute the cost of each variable (in order to select the variable with the highest cost). A X10 skeleton implementation of selectVarHighCost function is presented in Listing 1.2.

Listing 1.2. Function selVarHighCost in X10

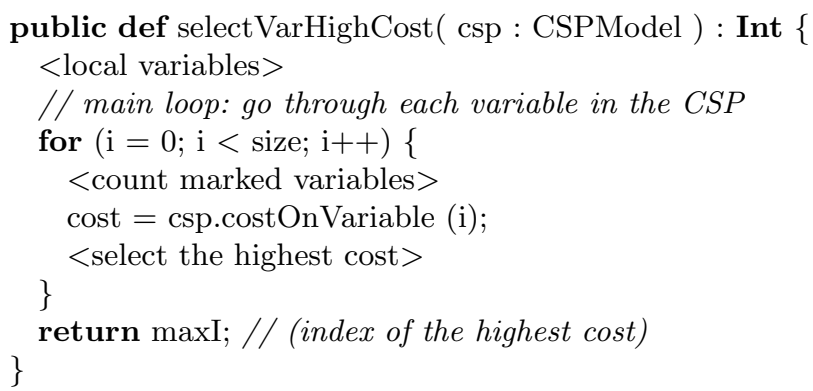

Since this function must process the entire variable vector at each iteration, it is natural to try to parallelize the task. For problems with many variables (e.g. the magic square problem involves $N^{2}$ variables) the gain could be very interesting. We developed a first approach (called FP1), in which $\mathrm{n}$ single activities are created at each iteration. Each activity processes a portion of the variables array and performs the required computations. The X10 construct async was chosen to create individual activities sharing the global array. Listing 1.3 shows the X10 skeleton code for the first approach of the functional parallelism in the function selectVarHighCost.

Listing 1.3. First approach to functional parallelism

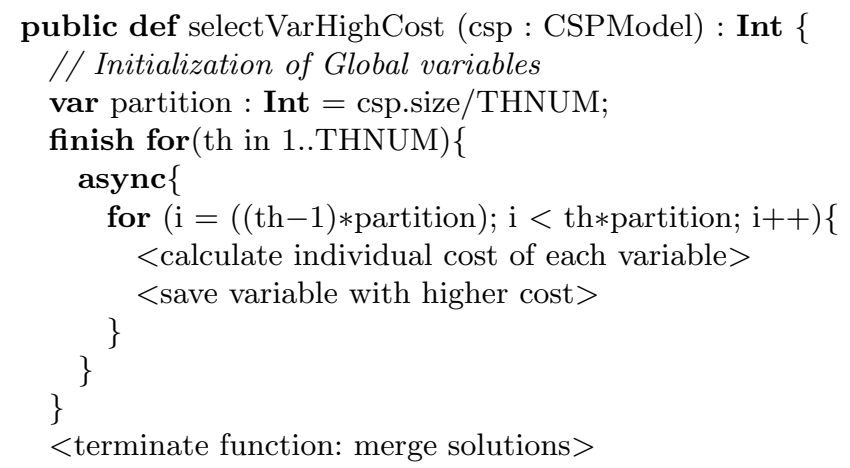


In this implementation the constant THNUM on line 4 represents the number of concurrent activities that are deployed by the program. On the same line, the keyword finish ensures the termination of all spawned activities. Finally, the construct async on line 5 spawns independent individual tasks to cross over a portion of the variable array (sentence for on line 6). With this strategy we face up with a well known problem of functional parallelism: the overhead due to the management of fine-grained activities. As expected results are not good enough (see Section 4 for detailed results).

In order to limit the overhead due to activity creation, we implemented a second approach (called FP2). Here the $n$ working activities are created at the very beginning of the solving process, just before the main loop of the algorithm. These activities are thus available for all subsequent iterations. However, it is necessary to develop a synchronization mechanism to assign tasks to the working activities and to wait for their termination. For this purpose we created two new classes: ComputePlace and ActivityBarrier. ComputePlace is a compute instance, which contains the functionality of the working activities. ActivityBarrier is a very simple barrier developed with X10 monitors (X10 concurrent package).

Listing 1.4 shows the X10 implementation of the second approach.

Listing 1.4. Second approach to functional parallelism

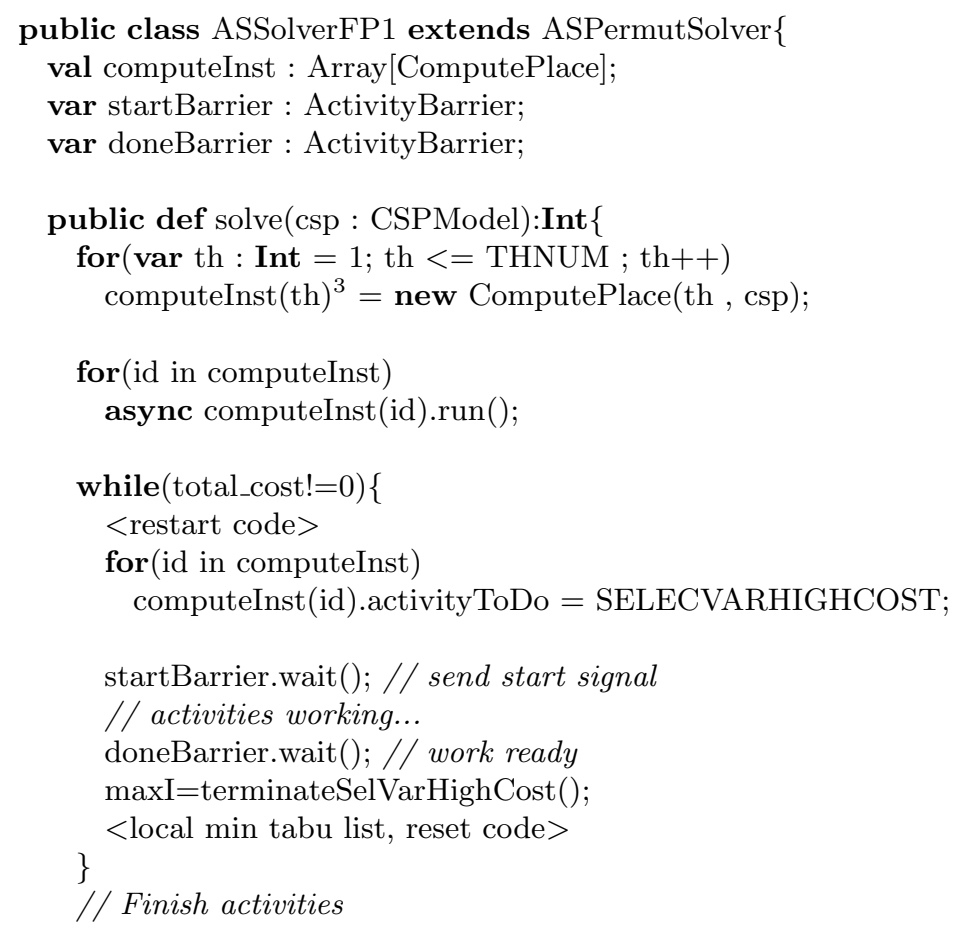

${ }^{3}$ Remark: in X10 the array notation is table(index) instead of table[index] as in C. 


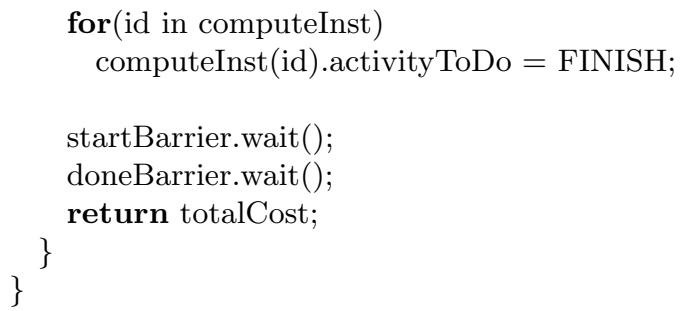

This code begins with the definition of three global variables on lines 24: computeInst, startBarrier and doneBarrier; computeInst is an array of ComputePlace objects, one for each working activity desired. startBarrier and doneBarrier are ActivityBarrier instances created to signalize the starting and ending of the task in the compute place. On lines 7-11, before the main loop THNUM working activities are created and started over an independent X10 activity. When the algorithm needs to execute the selectVarHighCost functionality, the main activity assigns this task putting a specific value into the variable activity ToDo in the corresponding instance of the ComputePlace class (lines 15 and 16), then the function wait() is executed over the barrier startBarrier to notify all working activities to start (line 18). Finally, the function wait() is executed over the barrier doneBarrier to wait the termination of the working activities (line 20). Then on line 21 the main activity can process the data with the function terminateSelVarHighCost. When the main loop ends, all the working activities are notified to end and the solve function returns (lines 25-30). Unfortunately, as we will see below, the improvement of this second approach is not important enough (and, in addition, it has its own overhead due to synchronization mechanisms).

\subsection{Data Parallel Implementation}

A straightforward implementation of data parallelism in the Adaptive Search algorithm is the multiple independent Independent Multi-Walks (IMW) approach. The idea is to use isolated sequential Adaptive Search solver instances dividing the search space of the problem through different random starting points. This strategy is also known as Multi Search (MPSS, Multiple initial Points, Same search Strategies) [5] and has proven to be very efficient [6,13].

The key of this implementation is to have several independent and isolated instances of the Adaptive Search Solver applied to the same problem model. The problem is distributed to the available processing resources in the computer platform. Each solver runs independently (starting with a random assignment of values). When one instance finds a solution it is necessary to stop all other running instances. This is achieved using a termination detection communication strategy. This simple parallel version has no inter-process communication, making it Embarrassingly or Pleasantly Parallel. The skeleton code of the algorithm is shown in the Listing 1.5.

Listing 1.5. Adaptive Search data parallel X10 implementation

1 public class ASSolverIMW\{ 


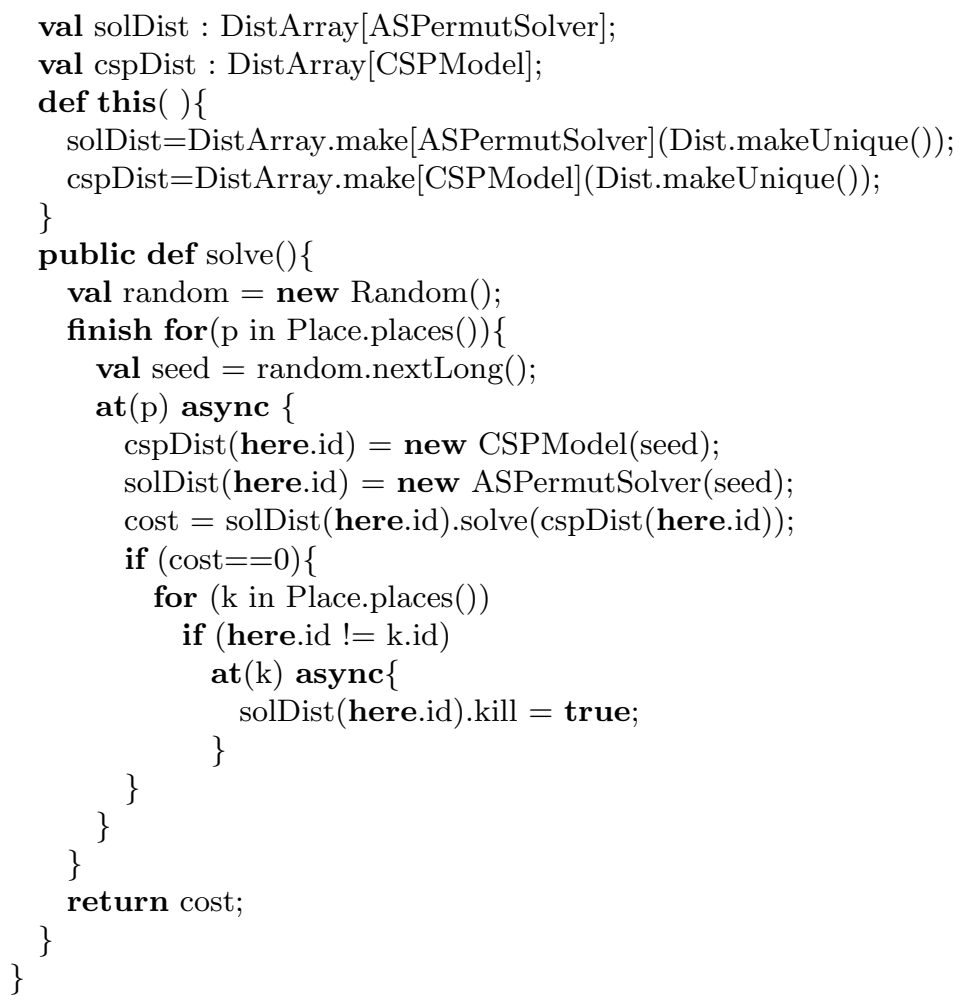

For this implementation the ASSolverIMW class was created. The algorithm has two global distributed arrays: solDist and cspDist (lines 2 and 3). As explained in Section 2, the DistArray class creates an array which is spread across multiple X10 places. In this case, an instance of ASPermutSolver and CSPModel are stored at each available place in the program. On lines 5 and 6 function make creates and initializes the distributed vector in the region created by the function Dist.makeUnique(). The makeUnique function creates a distribution over a region that maps every point in the region to a distinct place, and also maps some point in the region to every place. On line 10 a finish operation is executed over a for loop that goes through all the places in the program (Place.places()). Then, an activity is created in each place with the sentence at $(p)$ async on line 12. Into the async block, a new instance of the solver (new ASPermutSolver(seed)) and the problem (new CSPModel(seed)) are created (lines 13 and 14) and a random seed is passed. On line 15, the solving process is executed and the returned cost is assigned to the cost variable. If this cost is equal to 0 , the solver in a place has reached a valid solution, it is then necessary to send a termination signal to the remaining places (lines 16- 22). For this, every place (i.e. every solver), checks the value of a kill variable at each iteration. When it becomes equal to true the main loop of the solver is broken and the activity is finished. To set a kill remote variable from any X10 place it was necessary to create a new activity into each remaining place (sentence at(k) async on line 19) and into the async block 
to change the value of the kill variable. On line 18, the sentence if (here.id != $k$.id) filters all places which are not the winning one (here). Finally, the function returns the solution of the fastest place on line 25 .

\section{Performance Analysis}

We now present and discuss the experimental results of our X10 implementations of the Adaptive Search algorithm. The testing environment used was a nonuniform memory access (NUMA) computer, with 2 Intel Xeon W5580 CPUs each one with 4 hyper-threaded cores running at $3.2 \mathrm{GHz}$ as well as a system based on 4 16-core AMD Opteron 6272 CPUs running at $2.1 \mathrm{GHz}$.

We used a set of benchmarks composed of four classical problems in constraint programming: the magic square problem (MSP), the number partitioning problem (NPP) and the all-interval problem (AIP), all three taken from the CSPLib [8]; also we include the Costas Arrays Problem (CAP) introduced in [11], which is a very challenging real problem. The problems were all tested on significantly large instances which are generally out of reach of the traditional complete solvers like Gecode [22]. The interested reader may find more information on these benchmarks in [15].

It is worth noting, at the software level, that the X10 runtime system can be deployed in two different backends: a Java or a $\mathrm{C}++$ backend; they differ in the native language used to implement the X10 program (Java or $\mathrm{C}++$ ), also they present different trade-offs on different machines. Currently, the $\mathrm{C}++$ backend seems relatively more mature and faster for scientific computation, and therefore became our choice for this experimentation.

Regarding the stochastic nature of the Adaptive Search behavior, several executions of the same problem were done and the times averaged. We ran 100 samples for each experimental case in the benchmark.

In this presentation, all tables report raw times in seconds (average of 100 runs) and relative speed-ups. These tables respect the same format: the first column identifies the problem instance, the second column is the execution time of the problem in the sequential implementation, the next group of columns contains the corresponding speed-up obtained with a varying number of cores (places), and the last column presents the execution time of the problem with the highest number of places.

\subsection{Sequential Performance}

Even if our first goal in using X10 is parallelism, it is interesting to compare the sequential X10 implementation with a reference implementation: our low-level and highly optimized $\mathrm{C}$ version initially used in $[3,4]$ and continuously improved since then. With the latest versions, the X10 implementation appears to be 2 to 3 times slower than the $\mathrm{C}$ version, a consequence of the more complex runtime and the lack of optimization in the compiler. We feel that this is not a prohibitive 
price to pay, if one takes into account the possibilities promised by X10 for future experimentation.

A possible explanation of the difference between the performances of both implementations is probably the richness of the X10 language (OOP, architecture abstractions, communication abstractions, etc.). Also, maybe it is necessary to improve our X10 language skills good enough to get the best performance of this tool.

\subsection{Functional Parallel Performance}

Table 1 shows the results of the first version of the functional parallelism X10 implementation. Only two benchmarks (2 instances of MSP and CAP) are presented. Indeed, we did not investigate this approach any further since the results are clearly not good. Each problem instance was executed with a variable number of activities (THNUM $=2,4$ and 8 ). It is worth noting, that the environmental X10 variable X10_NTHREADS was passed to the program with an appropriate value to each execution. This variable controls the number of initial working threads per place in the X10 runtime system.

\begin{tabular}{||c|c|c|c|c|c||}
\hline Problem & time (s) & \multicolumn{2}{|c|}{ speed-up with k places } & time (s) \\
\cline { 3 - 6 } instance & seq. & 2 & 4 & 8 & 8 places \\
\hline \hline MSP-100 & 11.98 & 0.86 & 0.95 & 0.77 & 15.49 \\
MSP-120 & 24.17 & 1.04 & 0.97 & 0.98 & 24.65 \\
\hline CAP-17 & 1.56 & 0.43 & 0.28 & 0.24 & 6.53 \\
CAP-18 & 12.84 & 0.51 & 0.45 & 0.22 & 57.16 \\
\hline
\end{tabular}

Table 1. Functional Parallelism - first approach (timings and speed-ups)

As seen in Table 1, for all the treated cases the obtained speed-up is less than 1 (i.e. a slowdown factor), showing a deterioration of the execution time due to this parallel implementation. So, it is possible to conclude that no gain time is obtainable in this approach. To analyze this behavior it is important to return to the description of the Listing 1.3. As already noted, the parallel function selVarHighCost in this implementation are located into the main loop of the algorithm, so THNUM activities are created, scheduled and synchronized at each iteration in the program execution, being a very important source of overhead. The results we obtained suggest that this overhead is larger than the improvement obtained by the implementation of this parallel strategy.

Turning to the second approach, Table 2 shows the results obtained with this strategy. Equally, the number of activities spawn, in this case at the beginning, was varied from 2 to 8 .

Even if the results are slightly better, there is no noticeable speed-up. This is due to a new form of overhead tied to the synchronization mechanism which 


\begin{tabular}{||c|c|c|c|c|c||}
\hline Problem & time $(\mathrm{s})$ & \multicolumn{2}{|c|}{ speed-up with k places } & time $(\mathrm{s})$ \\
\cline { 3 - 6 } instance & seq. & 2 & 4 & 8 & 8 places \\
\hline \hline MSP-100 & 11.98 & 1.15 & 0.80 & 0.86 & 13.87 \\
MSP-120 & 24.17 & 1.23 & 0.94 & 0.63 & 38.34 \\
\hline CAP-17 & 1.56 & 0.56 & 0.30 & 0.25 & 6.35 \\
CAP-18 & 12.84 & 0.74 & 0.39 & 0.27 & 46.84 \\
\hline
\end{tabular}

Table 2. Functional Parallelism - second approach (timings and speed-ups)

is used in the inner loop of the algorithm, to assign tasks and wait for their termination (see Listing 1.4).

The performance model in X10 [9] specifies that the current implementation of async tasks in X10 has a considerable amount of overhead. Large number of fine grained async tasks are likely to decrease the performance of the application. Actually an implementation of Adaptive Search on GPU using CUDA language, reported by [1] shows that some performance improvement can be achieved, but activities have to be fine-tuned at a low level.

\subsection{Data Parallel Performance}

Table 3 and Figure 3 document the speedups we obtained when resorting to data parallelism. Observe that, for this particular set of runs, we used a different hardware platform, with more cores than for the other runs.

\begin{tabular}{||c|c|c|c|c|c|c||}
\hline Problem & time (s) & \multicolumn{4}{|c|}{ speed-up with k places } & time (s) \\
\cline { 3 - 7 } instance & seq. & 8 & 16 & 24 & 32 & 32 places \\
\hline \hline AIP-300 & 56.7 & 4.7 & 7.1 & 9.9 & 10.0 & 5.6 \\
NPP-2300 & 6.6 & 6.1 & 9.8 & 10.5 & 12.0 & 0.5 \\
MSP-200 & 365 & 8.3 & 12.2 & 13.6 & 14.6 & 24.9 \\
CAP-20 & 731 & 5.6 & 12.0 & 16.1 & 20.5 & 35.7 \\
\hline
\end{tabular}

Table 3. Data Parallelism (timings and speed-ups)

The performance of data parallel version is clearly above the performance of the functional parallel version. The resulting average runtime and the speedups obtained in the entire experimental test performed seems to lie within the predictable bounds proposed by [24]. The Costas Arrays Problem displays remarkable performance with this strategy, e.g. the CAP reaches a speed-up of 20.5 with 32 places. It can be seen that the speed-up increases almost linearly with the number of used places. However, for other problems (e.g. MSP), the curve clearly tends to flatten out when the number of places increases. 


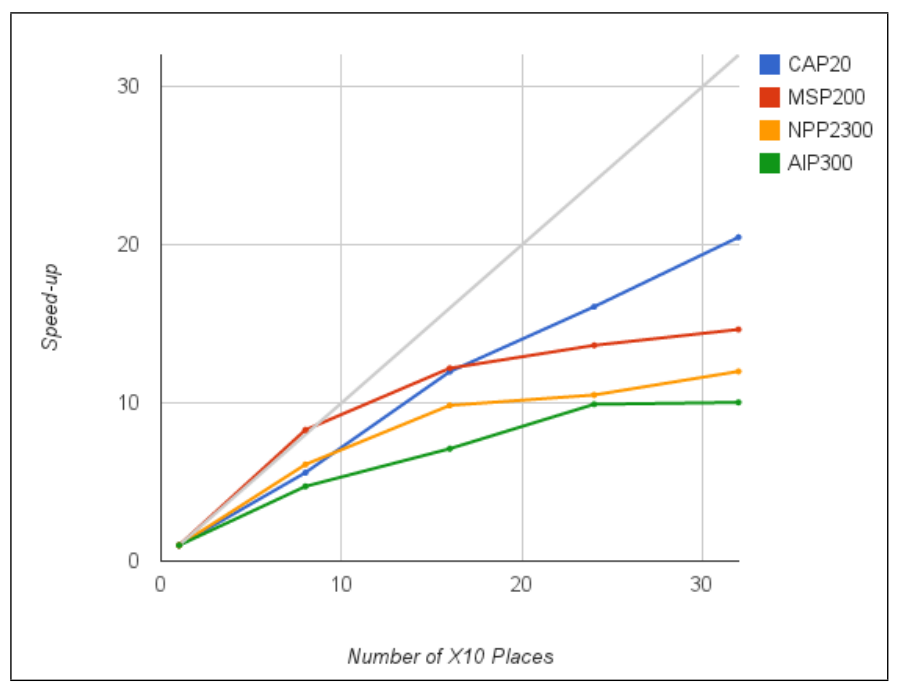

Fig. 3. Speed-ups for the most difficult instance of each problem

\section{Conclusion and Future Work}

We presented different parallel X10 implementations of an effective Local Search algorithm, Adaptive Search in order to exploit various sources of parallelism. We first experimented two functional parallelism versions, i.e. trying to divide the inner loop of the algorithm into various concurrent tasks. This turned out not to yield any speed-up at all, most likely because of the bookkeeping overhead (creation, scheduling and synchronization) that is incompatible with such a finegrained level of parallelism.

We then proceeded with a data parallel implementation, in which the search space is decomposed into possible different random initial configurations of the problem and getting isolated solver instances to work on each point concurrently. We got a good level of performance for the X10 data-parallel implementation with monotonously increasing speed-ups in all the problems we studied, although they taper off after some point.

The main result we draw from this experiment, is that X10 has proved a suitable platform to exploit parallelism in different ways for constraint-based local search solvers. These entail experimenting with different forms of parallelism, ranging from single shared memory inter-process communication to a distributed memory programming model. Moreover, the use of the X10 implicit communication mechanisms allowed us to abstract away from the complexity of the parallel architecture with a very simple and consistent device: the distributed arrays and the termination detection system in our data parallel implementation.

Considering that straightforward forms of parallelism seem to get lower gains as we increase the number of cores, we want to look for ways of improving on this situation. Future work will focus on the implementation of a cooperative Local 
Search parallel solver based on data parallelism. The key idea being to take advantage of the many communications tools available in the APGAS model, to exchange information between different solver instances in order to obtain a more efficient and, most importantly, scalable solver implementation. We also plan to test the behavior of a cooperative implementation under different HPC architectures, such as the many-core Xeon Phi, GPGPU accelerators and grid computing platforms.

\section{References}

1. Alejandro Arbelaez and Philippe Codognet. A GPU Implementation of Parallel Constraint-based Local Search. In 22nd Euromicro International Conference on parallel distributed and network-based processing, Turin, Italy, 2014.

2. David Butenhof. Programming With Posix Threads. Addison-Wesley Professional, 1997.

3. Philippe Codognet and Daniel Diaz. Yet another local search method for constraint solving. In Kathleen Steinhöfel, editor, Stochastic Algorithms: Foundations and Applications, pages 342-344. Springer Berlin Heidelberg, London, 2001.

4. Philippe Codognet and Daniel Diaz. An Efficient Library for Solving CSP with Local Search. In 5th international Conference on Metaheuristics, pages 1-6, Kyoto, Japan, 2003.

5. Teodor Gabriel Crainic and Michel Toulouse. Parallel Meta-Heuristics. In Michel Gendreau and Jean-Yves Potvin, editors, Handbook of Metaheuristics, number May, pages 497-541. Springer US, 2010.

6. Daniel Diaz, Salvador Abreu, and Philippe Codognet. Targeting the Cell Broadband Engine for constraint-based local search. Concurrency and Computation: Practice and Experience (CCPEE), 24(6):647-660, 2011.

7. Tarek El-Ghazawi, William Carlson, Thomas Sterling, and Katherine Yelick. Wiley: UPC: Distributed Shared Memory Programming - Tarek El-. Wiley, 2005.

8. I.P. Gent and T. Walsh. "CSPLib: a benchmark library for constraints. Technical report, 1999.

9. David Grove, Olivier Tardieu, David Cunningham, Ben Herta, Igor Peshansky, and Vijay Saraswat. A performance model for X10 applications: what's going on under the hood? In 2011 ACM SIGPLAN X10 Workshop, pages 1-8, San Jose, California, 2011. ACM.

10. Cray Inc. Chapel Language Specification, 2012. Also available as http://chapel. cray.com.

11. Serdar Kadioglu and Meinolf Sellmann. Dialectic Search. In Principles and Practice of Constraint Programming (CP), volume 5732, pages 486-500, 2009.

12. Khronos OpenCL Working Group. OpenCL Specification. 2008. Also available as https://www.khronos.org/opencl.

13. Rui Machado, Salvador Abreu, and Daniel Diaz. Parallel Local Search : Experiments with a PGAS-based programming model. In 12th International Colloquium on Implementation of Constraint and Logic Programming Systems, pages 1-17, Budapest, Hungary, 2012.

14. Rui Machado and Carsten Lojewski. The Fraunhofer virtual machine: a communication library and runtime system based on the RDMA model. Computer Science - $R E D D, 23(3-4): 125-132,2009$. 
15. Danny Múnera, Daniel Diaz, and Salvador Abreu. Experimenting with X10 for Parallel Constraint-Based Local Search. In Ricardo Rocha and Christian Theil Have, editors, Proceedings of the 13th International Colloquium on Implementation of Constraint and LOgic Programming Systems (CICLOPS 2013), August 2013.

16. NVIDIA. CUDA C Programming Guide, 2013. Also available as http://docs. nvidia.com/cuda/cuda-c-programming-guide.

17. OpenMP. The OpenMP API specification for parallel programming. Also available as http://openmp.org.

18. Francesca Rossi, Peter Van Beek, and Toby Walsh, editors. Handbook of Constraint Programming, volume 2 of Foundations of Artificial Intelligence. Elsevier Science, 2006.

19. Camille Salinesi, Raul Mazo, Olfa Djebbi, Daniel Diaz, and Alberto Lora-michiels. Constraints: the Core of Product Line Engineering. In Conference on Research Challenges in Information Science (RCIS), number ii, pages 1-10, Guadeloupe, French West Indies, France, 2011.

20. Vijay Saraswat, George Almasi, Ganesh Bikshandi, Calin Cascaval, David Cunningham, David Grove, Sreedhar Kodali, Igor Peshansky, and Olivier Tardieu. The Asynchronous Partitioned Global Address Space Model. In The First Workshop on Advances in Message Passing, pages 1-8, Toronto, Canada, 2010.

21. Vijay Saraswat, Bard Bloom, Igor Peshansky, Olivier Tardieu, and David Grove. X10 language specification - Version 2.3. Technical report, 2012. Also available as http://x10. sourceforge.net/documentation/languagespec/x10-latest.pdf.

22. C. Schulte, G. Tack, and M. Lagerkvist. Modeling and Programming with Gecode, 2013. Also available as http://www.gecode.org/.

23. Mark Snir, Steve Otto, Steven Huss-Lederman, David Walker, and Jack Dongarra. MPI : The Complete Reference. The MIT Press, 1996.

24. Charlotte Truchet, Florian Richoux, and Philippe Codognet. Prediction of parallel speed-ups for las vegas algorithms. In ICPP'2013, 42st International Conference on Parallel Processing, Lyon, France, October 1-4, 2013. IEEE Computer Society, 2013.

25. UPC Consortium, editor. UPC Language Specifications. 2005. Also available as http://upc.gwu.edu/docs/upc_specs_1.2.pdf. 


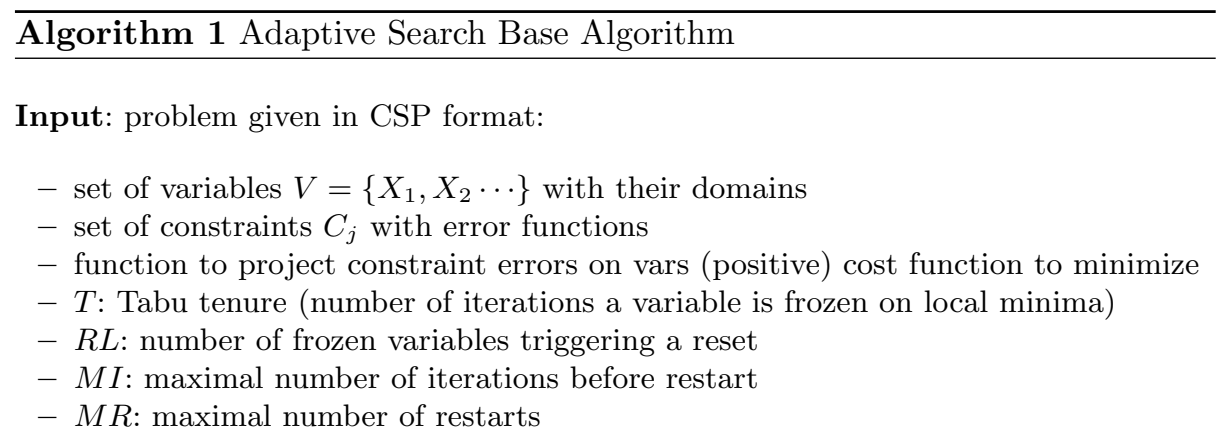

Output: a solution if the CSP is satisfied or a quasi-solution of minimal cost otherwise.

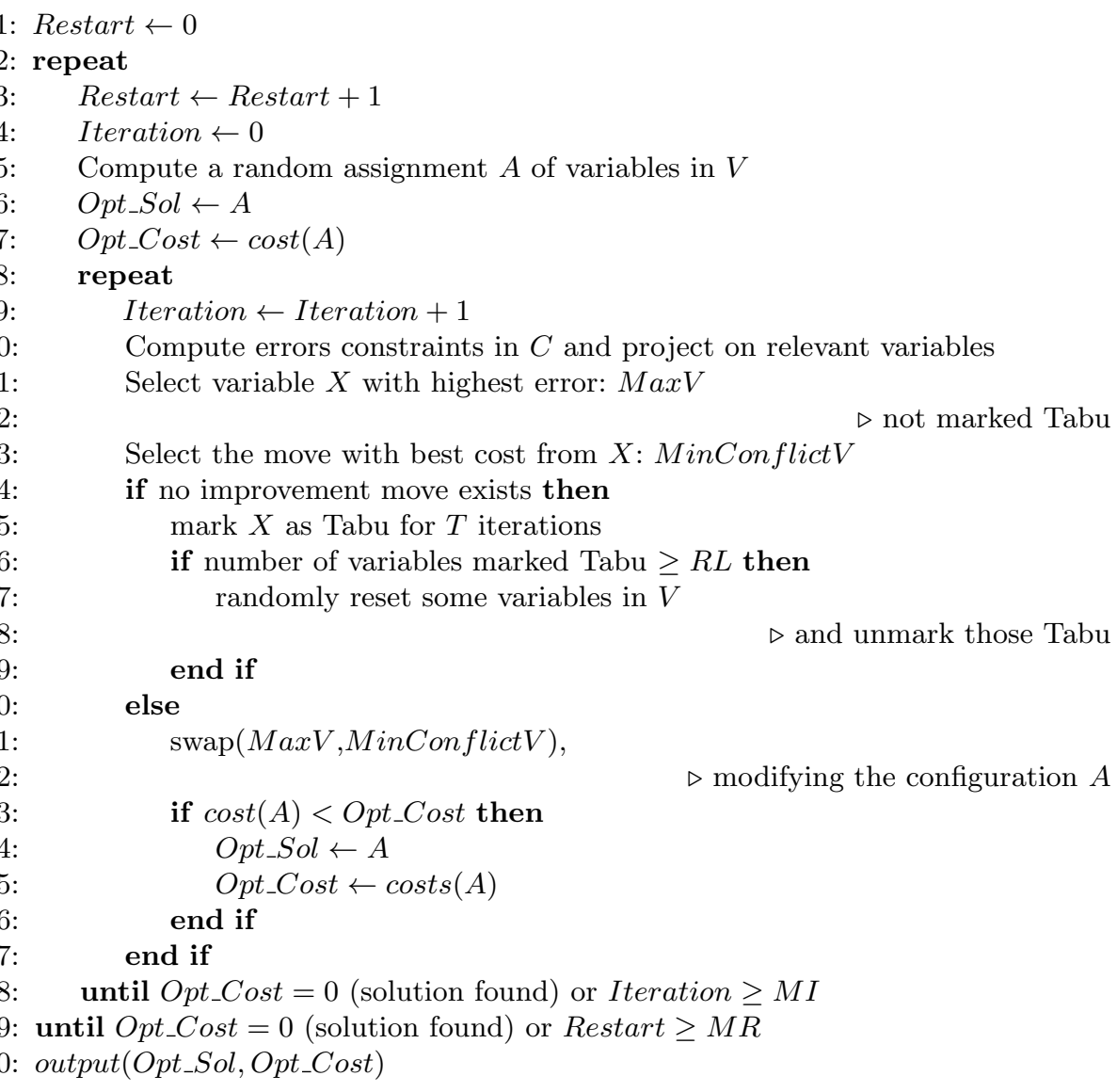

